\title{
CONHECIMENTOS DE USUÁRIOS DE UMA CLÍNICA CIRÚRGICA SOBRE A OCORRÊNCIA DE INCIDENTES
}

\author{
Ana Lúcia Queiroz Bezerra ${ }^{1}$, Thaynara de Oliveira Silva², Thatianny Tanferri de Brito Paranaguá ${ }^{3}$, Adrielle \\ Cristina Silva Souza ${ }^{4}$, Ana Elisa Bauer de Camargo Silva ${ }^{1}$, Cristiane Chagas Teixeira ${ }^{6}$
}

\begin{abstract}
RESUMO: A investigação objetivou identificar a ocorrência de incidentes percebidos pelos pacientes durante o período de internação hospitalar, analisar a opinião dos usuários sobre a ocorrência de incidentes e classificar os incidentes percebidos quanto ao tipo, causas e consequências. Estudo descritivo, transversal realizado por meio de entrevistas com 80 pacientes internados na clínica cirúrgica de um hospital de ensino, no ano de 2014, norteada por um instrumento validado. A exposição ao incidente foi percebida por $17,5 \%$ dos pacientes. Foram constatados 14 tipos de incidentes, sendo nove eventos adversos, quatro quase-erros e um incidente sem dano. Os mais perceptíveis ao paciente foram os decorrentes do processo de administração de medicamentos. Foi constatado que, apesar de inseridos no contexto da assistência, muitos profissionais ainda não reconhecem a importância do envolvimento dos usuários para a prevenção de incidentes, necessitando de ações educativas, com foco na segurança do paciente, para o empoderamento dos usuários.
\end{abstract}

DESCRITORES: Segurança do Paciente; Doença latrogênica; Enfermagem.

\section{KNOWLEDGE OF USERS OF A SURGERY CLINIC ABOUT THE OCURRENCE OF INCIDENTS}

\begin{abstract}
The aim of this study was to identify the occurrence of incidents that are noticed by patients during their hospital stay, to assess users views of the occurrence of incidents, and to classify noticed incidents as to type, causes, and consequences. This is a descriptive, cross-sectional study conducted through interviews with 80 patients admitted to the surgery clinic of a teaching hospital in 2014, guided by a validated instrument. Exposure to an incident was noticed by $17.5 \%$ of patients. Fourteen types of incidents were noticed, with nine being adverse events, four near-misses, and one an incident with no damage. The most noticeable incidents to the patient were those resulting from the medication administration process. Although within the context of care, many professionals have not yet recognized the importance of involving users to prevent incidents. In addition, educational activities focusing on patient safety for the empowerment of users are required.
\end{abstract}

DESCRIPTORS: Patient Safety; latrogenic Disease; Nursing.

\section{CONOCIMIENTOS DE PACIENTES DE UN SERVICIO DE CIRUGÍA SOBRE OCURRENCIA DE INCIDENTES}

RESUMEN: Se objetivó identificar la ocurrencia de incidentes percibidos por los pacientes durante su internación hospitalaria, analizar la opinión de los usuarios sobre ocurrencia de incidentes y clasificar los incidentes ocurridos según tipo, causas y consecuencias. Estudio descriptivo, transversal, realizado mediante entrevistas con 80 pacientes internados en servicio de cirugía de hospital de enseñanza en 2014, orientado por instrumento validado. La exposición al incidente fue percibida por 17,5\% de los pacientes. Fueron constatados 14 tipos de incidentes: nueve eventos adversos, cuatro cuasi-errores y un incidente sin consecuencias. Los más perceptibles para los pacientes fueron los derivados del proceso de administración de medicamentos. Fue constatado que, aún formando parte del ámbito de la atención, muchos profesionales aún no reconocen la importancia del compromiso de los usuarios en la prevención de incidentes, siendo necesarias acciones educativas enfocadas en la seguridad del paciente en pro del empoderamiento de los usuarios.

DESCRIPTORES: Seguridad del Paciente; Enfermedad latrogénica; Enfermería.

\footnotetext{
${ }^{1}$ Enfermeira. Doutora em Enfermagem. Docente de Enfermagem da Universidade Federal de Goiás. Goiânia, GO, Brasil.

${ }^{2}$ Enfermeira. Universidade Federal de Goiás. Goiânia, GO, Brasil.

${ }^{3}$ Enfermeira. Doutora em Enfermagem. Docente da Universidade de Brasília. Brasília, DF, Brasil.

${ }^{4}$ Enfermeira. Doutoranda em Enfermagem. Universidade Federal de Goiás. Goiânia,GO, Brasil.

${ }^{5}$ Enfermeira. Mestre em Enfermagem. Docente do Mais Sistema de Ensino. Alto Araguaia, MT, Brasil.
}

Autor Correspondente:

Ana Lúcia Queiroz Bezerra

Universidade Federal de Goiás

R. 227, s/n - 74605-080 - Goiânia, GO, Brasil

Email: analuciaqueiroz@uol.com.br
Recebido: 25/02/2016

Finalizado: $20 / 07 / 2016$ 


\section{INTRODUÇÃO}

Estima-se que de cada 10 pessoas que buscam assistência, pelo menos uma sofrerá agravos decorrentes de incidentes, os quais se definem como eventos ou circunstâncias advindas da assistência à saúde, não associados à doença de base, que poderiam resultar ou resultaram em dano ao paciente. Os incidentes são classificados em: circunstância notificável quando existe um potencial significativo para dano, mas o incidente não ocorreu; quase erro, quando o incidente não atingiu o paciente; incidente sem dano, quando o evento atingiu o paciente, mas não causou dano; e incidente com dano ou evento adverso, quando o incidente resulta em dano ao paciente ${ }^{(1)}$.

Estudo retrospectivo realizado na clínica cirúrgica de um hospital universitário apontou prevalência de $82 \%$ de incidentes sem dano e $18,7 \%$ de eventos adversos, sendo os mais frequentes aqueles relacionados ao processo clínico como omissão de cuidado, checagem inadequada e falhas durante procedimentos técnicos ${ }^{(2)}$.

Nessa perspectiva, foram criadas estratégias preventivas como o Programa Pacientes para a Segurança do Paciente, proposto pela Organização Mundial da Saúde (OMS), que situa os pacientes no centro dos cuidados e os torna parceiros plenos da assistência ${ }^{(1)}$. O programa Speak Up, elaborado pela Joint Comission International, também incentiva a participação do paciente durante o cuidado e tem a finalidade de orientar o paciente a esclarecer dúvidas sobre seu estado de saúde, tratamento, medicamentos em uso, documentos de consentimento sobre a assistência, assim como a eleger um familiar ou amigo para compartilhar as informações sobre o seu estado de saúde e para participar das decisões que envolvem o seu cuidado ${ }^{(3)}$.

Assim sendo, espera-se que os usuários estejam cada vez mais conscientes e participativos para o alcance da qualidade na prestação do cuidado seguro nos serviços de saúde.

No intuito de contribuir para a melhoria do cuidado à saúde e estimular o uso de estratégias que reduzam a ocorrência de incidentes, a partir do envolvimento do paciente no próprio cuidado, o presente estudo tem como objetivos analisar o conhecimento dos usuários sobre os incidentes ocorridos durante o período de internação hospitalar; classificar os incidentes referenciados, quanto ao tipo, causas e consequências.

\section{MÉTODO}

Estudo descritivo, transversal desenvolvido na Clínica Cirúrgica de um hospital de ensino. Participaram do estudo todos os pacientes internados no período de fevereiro a abril de 2014, que atenderam aos critérios de inclusão de possuir idade igual ou superior a 18 anos, estar em período pré e/ou pós-operatório, mentalmente orientado, com tempo de internação maior ou igual a 24 horas no momento da coleta de dados.

A coleta de dados foi por meio de entrevistas individuais, nos turnos diurnos, conforme disponibilidade do paciente. Foi utilizado um instrumento estruturado e validado composto por duas partes $^{(4)}$. A primeira com questões relacionadas às características sociodemográficas dos pacientes e aspectos clínicos da internação, e a segunda, de questões investigativas sobre a opinião dos pacientes quanto aos incidentes ocorridos durante a internação e sua participação na promoção da segurança durante o cuidado prestado pelos profissionais de saúde.

Os dados foram estruturados no software Statistical Package For The Social Science 20.0 e analisados descritivamente, apresentando médias e desvio padrão para variáveis contínuas e frequências relativas e absolutas para variáveis categóricas.

Os relatos sobre o incidente foram transcritos, analisados e, em seguida, julgados quanto ao tipo: circunstância notificável, quase erro, incidente sem dano e incidente com dano ou evento adverso, conforme o proposto pela OMS na Classificação Internacional para a Segurança do Paciente ${ }^{(1)}$. As consequências dos incidentes foram as decorrentes do atendimento prestado e relatadas pelos pacientes. 
O estudo está vinculado ao projeto: "Análise de ocorrências de eventos adversos em um hospital da Rede Sentinela da Região Centro Oeste", aprovado pelo Comitê de Ética em Pesquisa com Protocolo No 064/2008. Todos os pacientes foram esclarecidos sobre os objetivos do estudo e assinaram o termo de consentimento livre e esclarecido.

\section{RESULTADOS}

Participaram do estudo 80 pacientes, cujas características estão apresentadas na Tabela 1.

As comorbidades predominantes foram a hipertensão arterial e o diabetes melitus para, respectivamente, $45(56,5 \%)$ e $17(20,8 \%)$ dos pacientes.

Foi identificado que $65(81,3 \%)$ pacientes foram internados anteriormente, sendo 41 (51,3\%) neste hospital, sendo que 64 ( $80 \%$ ) foram encaminhados pelo sistema de regulação do Sistema Único de Saúde; 65 (81,3\%) foram admissões eletivas, com destaque para as especialidades cirurgia geral e vascular, com respectivamente $20(36,3 \%)$ e 12 $(15 \%)$.

O uso de pelo menos um dispositivo tubular foi relatado por 41 (52,5\%) dos pacientes, sendo que cinco $(6,3 \%)$ usaram sonda uretral, oito $(10 \%)$ dreno, $51(63,8 \%)$ acesso venoso periférico, três $(3,8 \%)$ cateter venoso central e quatro $(5 \%)$ sonda nasoentérica ou nasogástrica.

Dos pacientes, 72 (95\%) receberam algum tipo de informação na fase inicial do tratamento na clínica. Como informantes, o médico foi citado por $70(87,5 \%)$ e a enfermeira por apenas dois $(6 \%)$ dos pacientes.

Considerando outras fases do tratamento, 59 $(73,8 \%)$ informaram ter recebido pelo menos um tipo de informação sobre o preparo cirúrgico, quatro $(5 \%)$ receberam informações sobre os medicamentos prescritos, $28(35 \%)$ sobre o tratamento a ser realizado, $41(51,3 \%)$ sobre procedimentos realizados ou previstos e 20 (25\%) sobre exames.

Quanto ao conhecimento dos pacientes sobre incidentes ocorridos durante o período de internação, somente 14 (17,5\%) dos entrevistados relataram ter percebido algum incidente no período de sua internação. Desses pacientes, $11(78,5 \%)$ referiram incidentes relacionados ao processo de medicamentos com destaque para administração de medicamentos a paciente alérgico, erros de medicamento e de dose, omissão de dose e troca de paciente.

Do total de incidentes, sete $(50 \%)$ ocorreram no turno matutino. $O$ profissional envolvido
Tabela 1 - Características sociodemográficas dos pacientes internados na clínica cirúrgica de um hospital de ensino. Goiânia, GO, Brasil, 2014

\begin{tabular}{|c|c|c|}
\hline Características & $\mathbf{N}$ & $\%$ \\
\hline \multicolumn{3}{|l|}{ Sexo } \\
\hline Feminino & 49 & 61,3 \\
\hline Masculino & 31 & 38,8 \\
\hline \multicolumn{3}{|l|}{ Faixa etária } \\
\hline 19 a 29 anos & 11 & 13,8 \\
\hline 30 a 39 anos & 12 & 15 \\
\hline 40 a 49 anos & 20 & 20,5 \\
\hline 50 a 59 anos & 16 & 20 \\
\hline 60 a 69 anos & 13 & 16,3 \\
\hline 70 anos ou mais & 8 & 10 \\
\hline \multicolumn{3}{|l|}{ Estado Civil } \\
\hline Solteiro & 16 & 20 \\
\hline Casado & 44 & 55 \\
\hline Viúvo & 11 & 13,8 \\
\hline Outros & 9 & 11,3 \\
\hline \multicolumn{3}{|l|}{ Escolaridade } \\
\hline Fundamental Completo & 3 & 3,8 \\
\hline Fundamental Incompleto & 52 & 65 \\
\hline Médio Completo & 17 & 21,2 \\
\hline Médio Incompleto & 5 & 6,2 \\
\hline Superior Completo & 3 & 3,8 \\
\hline \multicolumn{3}{|l|}{ Procedência } \\
\hline Goiânia & 40 & 50 \\
\hline Interior de Goiás & 31 & 38,8 \\
\hline Outro estado & 9 & 11,3 \\
\hline \multicolumn{3}{|l|}{ Renda } \\
\hline Até 1 salário mínimo & 17 & 21,5 \\
\hline $\begin{array}{l}\text { Acima de } 1 \text { salário até } 3 \text { salários } \\
\text { mínimos }\end{array}$ & 31 & 38,4 \\
\hline Acima de 3 salários mínimos & 2 & 2,6 \\
\hline $\begin{array}{l}\text { Não soube informar os } \\
\text { rendimentos }\end{array}$ & 30 & 37,5 \\
\hline \multicolumn{3}{|l|}{ Possui comorbidades } \\
\hline Sim & 37 & 46,3 \\
\hline Não & 43 & 53,8 \\
\hline TOTAL & 80 & 100 \\
\hline
\end{tabular}


na maioria $12(85,7 \%)$ dos incidentes foi o técnico de enfermagem. Em relação às consequências decorrentes dos incidentes, sete (50\%) não causaram danos ao paciente, no entanto cinco $(35,7 \%)$ causaram danos e necessitaram de intervenção.

Para quatro (28,6\%) dos pacientes que sofreram incidentes, as causas prováveis foram distração, a fadiga e o estresse.

O Quadro 1 apresenta os relatos de nove pacientes sobre incidentes ocorridos durante a internação na unidade e relacionados à medicação.

Quadro 1 - Incidentes relacionados à medicação, percebidos pelos pacientes durante internação na clínica cirúrgica de um hospital de ensino. Goiânia, GO, Brasil, 2014

\begin{tabular}{|c|c|c|}
\hline $\begin{array}{l}\text { Incidente } \\
\text { (Classificação) }\end{array}$ & Circunstâncias em que ocorreu & Condutas / Causas \\
\hline $\begin{array}{l}\text { Reação alérgica } \\
\text { a medicamento. } \\
\text { (Evento } \\
\text { adverso) }\end{array}$ & $\begin{array}{l}\text { Administração de medicamento anestésico para } \\
\text { procedimento cirúrgico, que resultou em edema de } \\
\text { mucosa oral/labial e prolongamento da internação. }\end{array}$ & $\begin{array}{l}\text { Condutas: Suspensão da cirurgia. } \\
\text { Informado ao paciente sobre o evento. } \\
\text { Causas: Profissionais não investigaram } \\
\text { antecedentes alérgicos. }\end{array}$ \\
\hline $\begin{array}{l}\text { Reação alérgica } \\
\text { a medicamento. } \\
\text { (Evento } \\
\text { adverso) }\end{array}$ & $\begin{array}{l}\text { Administração de medicamento ao qual o paciente } \\
\text { era alérgico. Somente após sentir náuseas, tremor, } \\
\text { taquicardia e pico hipertensivo é que o paciente } \\
\text { informou à técnica de enfermagem que era alérgico } \\
\text { ao medicamento administrado. }\end{array}$ & $\begin{array}{l}\text { Condutas: Sem percepção de condutas. } \\
\text { Causas: Presença de profissionais } \\
\text { de outras unidades (rotatividade de } \\
\text { pessoal), que impediram melhor } \\
\text { investigação. }\end{array}$ \\
\hline $\begin{array}{l}\text { Troca de } \\
\text { medicamento. } \\
\text { (Quase-erro) }\end{array}$ & $\begin{array}{l}\text { Troca de soro entre pacientes, pela técnica } \\
\text { de enfermagem. Familiar viu que o rótulo do } \\
\text { frasco que seria infundido não era do paciente e } \\
\text { comunicou a profissional, interceptando a infusão. }\end{array}$ & $\begin{array}{l}\text { Condutas: A troca de soro foi corrigida. } \\
\text { Sem percepção de outras condutas. } \\
\text { Causas: Distração do profissional. }\end{array}$ \\
\hline $\begin{array}{l}\text { Troca de } \\
\text { medicamento. } \\
\text { (Quase-erro) }\end{array}$ & $\begin{array}{l}\text { Troca de soro entre pacientes, pela técnica } \\
\text { de enfermagem. Familiar viu que o rótulo do } \\
\text { frasco que seria infundido não era do paciente e } \\
\text { comunicou a profissional, interceptando a infusão. }\end{array}$ & $\begin{array}{l}\text { Condutas: A troca de soro foi corrigida. } \\
\text { Sem percepção de outras condutas. } \\
\text { Causas: Distração do profissional. }\end{array}$ \\
\hline $\begin{array}{l}\text { Troca de } \\
\text { medicamento } \\
\text { (Quase-erro). }\end{array}$ & $\begin{array}{l}\text { Falha na administração do medicamento dipirona, } \\
\text { pela técnica de enfermagem. Interceptação do } \\
\text { possível incidente pelo familiar por meio do rótulo. }\end{array}$ & $\begin{array}{l}\text { Condutas: A troca do medicamento } \\
\text { foi corrigida. Sem percepção de outras } \\
\text { condutas. } \\
\text { Causas: Falta de comunicação. }\end{array}$ \\
\hline $\begin{array}{l}\text { Omissão de } \\
\text { cuidado. } \\
\text { (Evento } \\
\text { adverso) }\end{array}$ & $\begin{array}{l}\text { Após administração de cinco gotas de Berotec, } \\
\text { paciente apresentou mal estar geral, queixando } \\
\text { desconforto cardiorrespiratório. Paciente } \\
\text { questionou a dose, mas foi informado que era } \\
\text { prescrição médica. Ausência de monitoramento de } \\
\text { reações a medicamentos, mesmo após reclamação } \\
\text { do paciente. Paciente não questionou profissional } \\
\text { de enfermagem por medo da reação do mesmo. } \\
\end{array}$ & $\begin{array}{l}\text { Conduta: Sem percepção de condutas. } \\
\text { Causas: Falta de conhecimento do } \\
\text { profissional sobre a dose máxima do } \\
\text { medicamento. } \\
\end{array}$ \\
\hline $\begin{array}{l}\text { Dose errada. } \\
\text { (Quase-erro) }\end{array}$ & $\begin{array}{l}\text { Administração de dose dupla de medicamento pela } \\
\text { técnica de enfermagem, que foi interceptado pelo } \\
\text { paciente ao ler, por acaso, o rótulo de identificação } \\
\text { do medicamento. Sem consequências mensuráveis. }\end{array}$ & $\begin{array}{l}\text { Conduta: Conferência da prescrição e } \\
\text { constatação do engano. } \\
\text { Causa: Sobrecarga de trabalho. }\end{array}$ \\
\hline $\begin{array}{l}\text { Dose errada. } \\
\text { (Evento } \\
\text { adverso) }\end{array}$ & $\begin{array}{l}\text { Administração de dose de insulina pela técnica de } \\
\text { enfermagem sem considerar o resultado do teste } \\
\text { de HGT, que foi de } 87 \mathrm{mg} / \mathrm{dl} \text {. Paciente apresentou } \\
\text { dormência na língua e visão turva. }\end{array}$ & $\begin{array}{l}\text { Condutas: Observação do paciente. } \\
\text { Causas: Distração do profissional. }\end{array}$ \\
\hline $\begin{array}{l}\text { Dose errada. } \\
\text { (Incidente sem } \\
\text { dano) }\end{array}$ & $\begin{array}{l}\text { Foi administrado dupla dosagem de um } \\
\text { medicamento ao paciente. Não foram percebidas } \\
\text { reações e/ou algum dano ao paciente. }\end{array}$ & $\begin{array}{l}\text { Condutas: Não perceptíveis ao } \\
\text { paciente. } \\
\text { Causas: Não conferência da dosagem } \\
\text { administrada com a folha de prescrição. }\end{array}$ \\
\hline
\end{tabular}


Entre os incidentes relacionados à medicação, foram detectados quatro eventos adversos, quatro quase-erros e um incidente sem dano. As consequências principais dos eventos adversos foram edema de mucosa oral/labial; prolongamento da internação, desconforto cardiorrespiratório, náuseas, tremor, taquicardia e pico hipertensivo, dormência na língua e visão turva. Os incidentes detectados pelos pacientes foram atribuídos à distração profissional, à falta de comunicação, à sobrecarga de trabalho e à alta rotatividade de profissionais nos locais de cuidado.

O Quadro 2 apresenta os relatos de cinco pacientes sobre os incidentes percebidos relacionados ao processo clínico durante a internação na unidade.

Os incidentes relacionados ao processo clínico foram $100 \%$ dos casos classificados como eventos adversos, sendo apontadas como as principais consequências a dor, formigamento e dormência, hemorragia esclerótica, gengivas, epistaxe e melena. $\mathrm{O}$ paciente foi quem detectou todas as falhas, apontando como causas a falta de habilidade profissional, a sobrecarga de trabalho e a falha durante a prescrição de medicamento.

Em relação ao acidente com o paciente, foi relatada apenas uma queda, que resultou em cefaleia intensa, sem abordagem profissional para análise da situação do paciente após o incidente.

Do total de 14 incidentes ocorridos, em 12 (87\%) o paciente e familiar foram os principais agentes de identificação de falhas e interceptação dos incidentes, especialmente os quase-erros, que corresponderam a quatro $(28,5 \%)$ das ocorrências. As condutas clínicas adotadas pelos profissionais para correção de falhas foram em consonância com o tipo de incidente. Entretanto, do universo de incidentes ocorridos, somente em seis $(42,9 \%)$ casos, os pacientes perceberam as condutas adotadas.

Quadro 2 - Incidentes relacionados ao processo clínico, percebidos pelos pacientes durante a internação na clínica cirúrgica de um hospital de ensino. Goiânia, GO, Brasil, 2014

\begin{tabular}{|l|l|l|}
\hline $\begin{array}{l}\text { Incidente } \\
\text { (Classificação) }\end{array}$ & Circunstâncias em que ocorreu & Condutas / Causas \\
\hline $\begin{array}{l}\text { Falha durante } \\
\text { procedimentos } \\
\text { invasivos. } \\
\text { (Evento adverso) }\end{array}$ & $\begin{array}{l}\text { Paciente relatou punção venosa pela técnica de } \\
\text { enfermagem sem sucesso e com complicações. } \\
\text { Apresentou dor, formigamento e dormência em } \\
\text { antebraço direito. }\end{array}$ & $\begin{array}{l}\text { Condutas: Não Houve. } \\
\text { Causas: Falta de habilidade do } \\
\text { profissional. }\end{array}$ \\
\hline $\begin{array}{l}\text { Omissão de } \\
\text { cuidado. } \\
\text { (Evento adverso) }\end{array}$ & $\begin{array}{l}\text { Paciente em pós-operatório imediato, proibida } \\
\text { de andar por 30 dias, solicitou auxílio à técnica } \\
\text { de enfermagem para ir ao banheiro, mas não foi } \\
\text { atendida. Ao levantar sozinha, caiu sobre as lixeiras } \\
\text { do quarto. Referiu dor persistente em membros } \\
\text { inferiores. }\end{array}$ & $\begin{array}{l}\text { Condutas: Não Houve. } \\
\text { Causas: Sobrecarga de trabalho. }\end{array}$ \\
\hline $\begin{array}{l}\text { Omissão de } \\
\text { cuidado. } \\
\text { (Evento adverso) }\end{array}$ & $\begin{array}{l}\text { Queixa de cefaleia e identificado elevação dos } \\
\text { níveis pressóricos pela técnica de enfermagem que } \\
\text { solicitou avaliação médica, mas não foi atendida. } \\
\text { Paciente continuou com cefaleia e apresentou } \\
\text { desconforto e ansiedade. }\end{array}$ & $\begin{array}{l}\text { Condutas: Observação do paciente. } \\
\text { Nauncia informado sódica. } \\
\text { Causas: Paciente não soube identificar } \\
\text { as causas. }\end{array}$ \\
\hline $\begin{array}{l}\text { Dose errada. } \\
\text { (Evento adverso) }\end{array}$ & $\begin{array}{l}\text { Paciente em pós-operatório tardio apresentou } \\
\text { hemorragia após iniciar o uso de anticoagulante, } \\
\text { com dose superior à necessária, seguindo com } \\
\text { quadro de hemorragia esclerótica, gengivas, } \\
\text { epistaxe e melena. }\end{array}$ & $\begin{array}{l}\text { Condutas: Paciente encaminhada para } \\
\text { a UTI. } \\
\text { Causas: Erro de prescrição. }\end{array}$ \\
\hline $\begin{array}{l}\text { Queda. } \\
\text { (Evento adverso) }\end{array}$ & $\begin{array}{l}\text { Paciente refere que levantou para ir ao banheiro } \\
\text { e caiu, batendo com a cabeça no chão e iniciando } \\
\text { cefaleia intensa. }\end{array}$ & $\begin{array}{l}\text { Condutas: Nenhuma. } \\
\text { Causas: falta de auxílio da equipe de } \\
\text { enfermagem. }\end{array}$ \\
\hline
\end{tabular}


Os incidentes são decorrentes de fatores organizacionais e podem estar relacionados aos membros da equipe de saúde ou constituir em fator individual do paciente e, em grande parte, são considerados evitáveis ${ }^{(1)}$.

Nesse estudo, o perfil dos usuários se assemelha aos resultados de um estudo retrospectivo realizado em uma clínica cirúrgica onde predominou o sexo feminino e a faixa etária adultos jovens, o que pode ser uma característica de internações cirúrgicas em hospital ${ }^{(2)}$.

O ensino fundamental incompleto e a renda mensal baixa, relatados pela maioria dos pacientes, podem estar relacionados ao grau de entendimento do indivíduo, às orientações recebidas pela equipe de saúde, sendo que pessoas com maior renda mensal têm maior grau de escolaridade e informação e, consequentemente, entendem a necessidade de hábitos saudáveis de saúde ${ }^{(5,6)}$. Neste estudo apesar do nível de instrução, os pacientes apresentaram entendimento das informações recebidas acerca do tratamento, além de clara percepção sobre a ocorrência de incidentes na prestação de cuidados pela equipe de saúde.

Nesse sentido, durante a European Conference on Patient, foi recomendado aos profissionais de saúde ouvir os usuários e educá-los para pensar criticamente e agir com autonomia, auxiliando-o a compreender o significado do seu papel nas ações de segurança do próprio cuidado ${ }^{(7)}$. Para promoção da sua saúde, pacientes empoderados interagem de forma mais eficaz com os profissionais de saúde tentando realizar ações que produzam melhores resultados ${ }^{(5)}$.

A comunicação efetiva possibilita um relacionamento positivo entre a equipe de saúde e o paciente, sendo fundamental para prestar uma boa assistência, bem como contribui para uma melhor e mais rápida recuperação do paciente ${ }^{(8)}$.

Uma das estratégias de prevenção de incidentes durante a hospitalização é a comunicação eficaz entre paciente/profissional, assim como o envolvimento do paciente no cuidado, considerando o mesmo como parceiro pleno na assistência. Portanto, a falta de sintonia na comunicação é fator de risco para a ocorrência de danos ao paciente ${ }^{(2,9)}$.

Dessa forma, se faz necessário que os profissionais de saúde reconheçam a importância do empoderamento do paciente e familiar e, ainda, desenvolvam ações educativas para que eles possam compreender a relevância, legitimidade e efetividade que suas próprias intervenções podem proporcionar ao seu cuidado ${ }^{(10)}$.

No contexto da assistência perioperatória, estima-se que, anualmente, sejam realizadas 234 milhões de cirurgias, e em decorrência desses procedimentos, ocorram dois milhões de óbitos e sete milhões de pessoas apresentam incidentes, sendo 50\% evitáveis. Dentre as cirurgias de alta complexidade, cerca de $3 \%$ a $16 \%$ registram incidentes com danos graves ${ }^{(1)}$.

O tipo de incidente mais prevalente foi relacionado à administração de medicamento. Estudo realizado em uma clínica cirúrgica apontou prevalência de $48 \%$ de incidentes relacionados à administração de medicamentos. No mesmo estudo, a omissão de dose foi a mais frequente. Os fatores de risco para a ocorrência de incidentes com medicamentos foram o tempo de internação prolongado, a realização de intervenção cirúrgica e o uso de múltiplas medicações ${ }^{(11)}$.

Na prática em saúde, uma das competências do profissional de enfermagem é informar ao paciente sobre o medicamento que será administrado, dose e via, e que isto é válido na redução de erros para pacientes orientados, conscientes e em bom estado geral.

No entanto, há evidência que os pacientes estão mais participativos na sua terapia medicamentosa, reconhecem o medicamento pela cor, formato e consistência, contribuindo assim na prevenção de erros, e nos faz refletir sobre a responsabilidade deles sobre sua terapia medicamentosa ${ }^{(12)}$.

O período matutino se apresenta como o mais propício para a ocorrência de incidentes, o que está associado ao modo de organização do trabalho, já que as atividades da clínica são concentradas nesse período. Essa é uma realidade encontrada em outro estudo em que o maior número de procedimentos 
ocorre no período matutino, e um dos procedimentos mais realizados é a administração de medicamentos $^{(13)}$.

O técnico de enfermagem foi referido como o principal profissional envolvido nos incidentes. A enfermagem é considerada a linha de frente para a administração de medicamentos, sendo uma das principais ações da equipe de enfermagem, que deve conhecer todos os aspectos e fases envolvidas no processo de administração de medicamentos a fim de prevenir danos ao paciente ${ }^{(12)}$.

A administração de medicamentos constitui-se em atividade complexa, no entanto, em decorrência do dimensionamento de pessoal inadequado, essa atividade, na maioria das instituições de saúde, é assumida pelo técnico de enfermagem com baixa supervisão do enfermeiro.

Dos incidentes identificados, a maioria foi classificado como evento adverso, ou seja, incidentes que atingiram o paciente e que causaram dano. Pelos relatos destes pacientes, foi possível identificar falha na comunicação profissional/paciente, a falta de atenção e consideração do profissional sobre o que o paciente está informando, e até a sobrecarga de trabalho dos profissionais.

A sobrecarga de trabalho do enfermeiro influencia na qualidade da assistência prestada aos pacientes, e estes percebem que a baixa qualidade do atendimento oferecido por este profissional está ligada ao grande número de atividades que esse precisa desenvolver ${ }^{(14)}$.

Quatro incidentes foram classificados como quase-erro, quando o incidente não atinge o paciente. O paciente foi quem detectou e alertou ao profissional quanto ao provável incidente, sendo a maioria relacionada à troca de medicamento. Pacientes e acompanhantes desempenham papel central no uso seguro de medicamentos. O envolvimento e a participação no cuidado implicam em prestar informações, tirar dúvidas e utilizar a medicação conforme a prescrição(15).

Nesta perspectiva, ressalta-se a importância do envolvimento do paciente durante sua assistência, de forma a direcionar seu próprio cuidado visando minimizar a ocorrência de incidentes, tornando-se a principal estratégia de prevenção dos $\operatorname{mesmos}^{(7)}$.

O Programa Paciente para a Segurança do Paciente, com intuito de incentivar que os pacientes sejam colocados no centro dos cuidados e incluídos como parceiros da assistência, tem estimulado as instituições a aderirem a essa prática pela segurança no cuidado ${ }^{(2)}$.

Estudo realizado por meio de grupo focal com pacientes e equipe de enfermagem em um hospital terciário no leste do Canadá, evidenciou que tanto o paciente quanto os profissionais de saúde admitem a importância de construir uma conexão pessoal para assegurar o envolvimento dos pacientes no seu cuidado e segurança. Entretanto, referem que o estresse e a carga de trabalho da equipe de enfermagem reduzem a capacidade dessa conexão, impossibilitando o processo de envolvimento do paciente $\mathrm{p}^{(16)}$.

Pesquisadores ressaltam que o incentivo por parte dos profissionais aumenta o interesse do paciente no envolvimento do seu cuidado e segurança, e que o paciente descreve que o engajamento com a segurança constitui um processo de aprendizagem, que inclui ser proativo, questionar e relatar qualquer desvio observado na rotina ${ }^{(17,18)}$.

O paciente deve participar da notificação de eventos adversos, pois ele é capaz de reconhecer detalhes que os membros da equipe não perceberam. Entretanto, apenas a notificação é insuficiente. Deve-se incentivar o uso de sistemas de informação, a fim de aprimorar o acompanhamento dos incidentes, a análise das tendências e a identificação das causas ${ }^{(19)}$.

Também há necessidade de melhorias na gestão dos processos de trabalho e do sistema organizacional, visando à construção de conhecimentos e atitudes para a prevenção de eventos adversos e promoção da cultura de segurança ${ }^{(20)}$.

Os resultados desse estudo reforçam a necessidade de educar os pacientes para a prevenção de incidentes, sendo que as ações educativas devem ser de responsabilidade da instituição de saúde ${ }^{(21)}$.

A literatura sobre a participação do paciente ainda é escassa. Entretanto, é possível constatar nuances do despertar de pacientes e profissionais para esse novo desafio que está associado ao desenvolvimento de uma cultura institucional e social no âmbito da segurança e qualidade dos serviços de saúde. 
A realidade destacada neste estudo aponta sobre a importância da temática "paciente para a segurança do paciente", assunto ainda pouco explorado pela comunidade científica.

Enquanto o paciente está atento a tudo que envolve o cuidado que lhe é prestado, se interessa e se insere no contexto de sua assistência, muitos profissionais ainda não reconhecem a importância dessa parceria para a prevenção de incidentes e, consequentemente, a melhoria da qualidade em saúde.

Desta forma, a participação dos usuários na segurança do cuidado durante a internação fica comprometida, fazendo-se necessária a elaboração de ações educativas, visando à capacitação dos profissionais de saúde com foco na segurança do paciente, ressaltando a utilização da comunicação e empoderamento dos usuários dos serviços de saúde como estratégias de prevenção de incidentes.

Considera-se como limitação, o fato do estudo ter sido desenvolvido em uma Clínica Cirúrgica, restringindo o campo a uma instituição hospitalar e a uma população específica, limitando os resultados encontrados a grupos similares, sendo necessário o investimento em outras pesquisas para confirmar os resultados encontrados. Entretanto, o presente estudo apresenta compreensões a partir da análise de uma determinada realidade e subjetividade de cada paciente, visto que apresentavam níveis diversos de complexidade assistencial, assim como educacional, o que pode ter influenciado nas respostas. Pesquisar o conhecimento de pacientes na perspectiva da segurança durante o processo assistencial pode servir como estratégia para influenciar na melhoria do nível de qualidade do cuidado prestado.

\section{REFERÊNCIAS}

1. World Health Organization (WHO). Patients for Patient Safety: forward program [Internet]. Genève; 2004 [citado em 05 jan 2016]. Disponível: http://www.who.int/patientsafety/patients_for_patient/en/

2. Paranaguá TTB, Bezerra ALQ, Silva AEBC, Filho FMA. Prevalência de incidentes sem dano e eventos adversos em uma clínica cirúrgica. Acta paul. enferm. 2013; 26(3): 256-62.

3. Joint commission on the accreditation of healthcare organizations. "Speak Up" [Internet]. Illinóis. 2005 [acesso em 15 jan 2016]. Disponível: http://www.jointcommission.org/assets/1/18/speakup_amb.pdf

4. de Freitas JS, Silva AEBC, Minamisava R, Bezerra ALQ, de Sousa MRG. Qualidade dos cuidados de enfermagem e satisfação do paciente atendido em um hospital de ensino. Rev. Lat-Am. Enferm. 2014, 22(3): 454-60.

5. Taddeo PS, Gomes KWL, Caprara A, Gomes AMA, de Oliveira GC, Moreira TMM. Acesso, prática educativa e empoderamento de pacientes com doenças crônicas. Ciên.saúde coletiva. 2012; 17(11): 2923-30.

6. dos Santos AMA, Jacinto PA, Tejada CAO. Causalidade entre renda e saúde: uma análise através da abordagem de dados em painel com os Estados do Brasil. Est. Econ. 2012; 42(2): 229-61.

7. Silva GS. Empoderar o paciente. O que é isso? Saúde Business. [Internet] 2014 [acesso em 17 fev. 2016]. Disponível: http://saudebusiness.com/noticias/empoderar-o-paciente-o-que-e-isso/

8. Gaspar MRF, Massi GA, Gonçalves CGO, Willig MH. A equipe de enfermagem e a comunicação com o paciente traqueostomizado. Rev. CEFAC. [Internet] 2015; 17(3) [acesso em 18 fev 2016]. Disponível:

http://dx.doi.org/10.1590/1982-0216201514214

9. Dornfeld D, Pedro ENR. A comunicação como fator de segurança e proteção ao parto. Rev. Eletr. Enf. [Internet] 2011; 13(2) [acesso em 18 fev 2016]. Disponível: http://dx.doi.org/10.5216/ree.v13i2.10925

10. Longtin Y, Sax H, Leape LL, Sheridan SE, Donaldson L, Pittet D. Patient participation: Current knowledge and apllicability to patient safety. Mayo Clin Proc. 2010; 85(1): 53-62.

11. Paranaguá TTB, Bezerra ALQ, Santos ALM, Silva AEBC. Prevalência e fatores associados aos incidentes relacionados à medicação em pacientes cirúrgicos. Rev Esc Enferm USP. 2014; 48(1): 41-8. 
12. Franco JN, Ribeiro G, D'Innocenzo M, Barrros BPA. Percepção da equipe de enfermagem sobre fatores causais de erros na administração de medicamentos. Rev Bras Enferm. 2010; 63(6): 927-32.

13. Prates DO. Análise das interrupções ocorridas durante a assistência de enfermagem em unidades de tratamento intensivo [dissertação]. Goiânia: Universidade Federal de Goiás - UFG; 2015.

14. Wisniewski D, Gróss G, Bittencourt R. A influência da sobrecarga de trabalho do enfermeiro na qualidade da assistência pré-natal. Rev Bras Promoç Saúde, 2014; 27(2): 177-82.

15. Rede Brasileira de Enfermagem e Segurança do Paciente. Estratégias para a segurança do paciente: manual para profissionais da saúde / Rede Brasileira de Enfermagem e Segurança do Paciente. - Porto Alegre: EDIPUCRS, 2013.

16. Bishop AC, Macdonald M. Patient Involvement in patient safety: a qualitative study of nursing staff and patient perceptions. J Patient Saf. [Internet] 2014; 10(3) [acesso em 18 fev 2016]. Disponível:

http://dx.doi.org/10.1097/pts.0000000000000123

17. Davis RE, Sevdalis N, Vincent CA. Patient involvement in patient safety: how willing are patients to participate? BMJ Qual Saf. 2011; 20: 108-14.

18. Schwappach DLB. Review: engaging patients as vigilant partners in safety - a systematic review. Med Care Res Rev. 2010; 67(2): 119-48.

19. King A, Daniels J, Lim J, Cochrane DD, Taylor A, Ansermino JM. Time to listen: review of methods to solicit patient reports of adverse events. Qual Saf Health Care. 2010; 19(2): 148-57.

20. Moreira IA, Bezerra ALQ, Paranaguá TTB, Silva AEBC, Filho FMA. Conhecimento dos profissionais de saúde sobre eventos adversos em unidade de terapia intensiva. Rev enferm UERJ. 2015; 23(4): 461-7

21. Schwappach DLB. Patients and healthcare workers perceptions of a patient safety advisory. Int J Qual Health Care. 2011; 23(6): 1713-20. 